Oscar Blasco, Pablo Gregori and Jose M. Calabuig*, Departamento de Análisis Matemático, Universidad de Valencia, 46100 Burjassot, Valencia, Spain. e-mail: oscar.blasco@uv.es, pablo.gregori@uv.es,

jose.m.calabuig@uv.es

\title{
FINITE SEMIVARIATION AND REGULATED FUNCTIONS BY MEANS OF BILINEAR MAPS
}

\begin{abstract}
It is shown that $Z$ does not contain a copy of $c_{0}$ if and only if for every bilinear map $B: X \times Y \rightarrow Z$, every function $f:[0,1] \rightarrow X$ of bounded $(\mathcal{B})$-variation is $(\mathcal{B})$-regulated.
\end{abstract}

\section{Introduction}

In the setting of Riemann-Stieltjes type integrals, and more precisely in the Henstock-Kurzweil or Dushnik or Young integrals, the concepts of 'function of bounded variation' and 'regulated function' defined by means of a particular bilinear form play an important role. In a recent paper [1], L. Barbanti established the relationship between the two concepts under a uniform convexity assumption for some particular bilinear forms. In this note we present the assumption which characterizes this relationship.

We shall work with functions $f$ defined over the unit interval $[0,1]$ and having values in a Banach space $X$, and we use the following notation: $D$ is the set of all finite partitions of $[0,1], d$ represents any element of $D$, and $|d|$ is the length of $d$; that is, $d=\left\{t_{0}, t_{1}, \ldots, t_{|d|}\right\}$ where $0=t_{0}<t_{1}<\ldots<t_{|d|}=1$.

First of all we recall the well-known definitions of the (total) variation and

Key Words: bilinear triples, regulated functions, semivariation

Mathematical Reviews subject classification: 26A39

Received by the editors February 22, 2000

*All three authors supported by grant DGESIC PB98-1426 
semivariation of a function

$$
\begin{aligned}
& \operatorname{var}(f)=\sup _{d \in D} \sum_{k=1}^{|d|}\left\|\left[f\left(t_{k}\right)-f\left(t_{k-1}\right)\right]\right\|_{X} \\
& \operatorname{semivar}(f)=\sup _{d \in D}\left\|\sum_{k=1}^{|d|} \epsilon_{k}\left[f\left(t_{k}\right)-f\left(t_{k-1}\right)\right]\right\|_{X} \\
& =\sup _{\substack{d \in D \\
\left\|x^{*}\right\| \leq 1}} \sum_{k=1}^{|d|}\left|\left\langle f\left(t_{k}\right)-f\left(t_{k-1}\right), x^{*}\right\rangle\right|
\end{aligned}
$$

where $x^{*} \in X^{*}$

A function $f:[0,1] \rightarrow X$ is said to have finite variation when $\operatorname{var}(f)<\infty$, finite semivariation if semivar $(f)<\infty$ and to be regulated if it has at most discontinuities of first kind at every $t \in[0,1]$.

Let us consider now $\mathcal{B}=(B ; X, Y, Z)$ formed by three Banach spaces $X$, $Y$ and $Z$, and a continuous bilinear map $B: X \times Y \rightarrow Z$. We can extend now the concepts of variation and regulated functions as follows. A function $f:[0,1] \rightarrow X$ is said to have finite $(\mathcal{B})$-variation if

$$
(\mathcal{B}) \operatorname{var}(f):=\sup _{\substack{d \in D \\\left\|y_{k}\right\| \leq 1 \\ 1 \leq k \leq|d|}}\left\|\sum_{k=1}^{|d|} B\left(f\left(t_{k}\right)-f\left(t_{k-1}\right), y_{k}\right)\right\|_{Z}
$$

is finite, and it is called $(\mathcal{B})$-regulated if the function $f_{B, y}:[0,1] \rightarrow Z$ mapping $t \mapsto B(f(t), y)$ is regulated for every $y$ in the unit ball of $Y$. Let us mention the following three examples.

- $\mathcal{B}_{1}=\left(B_{1} ; X, \mathbb{K}, X\right)$ with $B_{1}(x, \lambda)=\lambda x$. Here $(\mathcal{B}) \operatorname{var}(f)=\operatorname{semivar}(f)$.

- $\mathcal{B}_{2}=\left(B_{2} ; X, X^{*}, \mathbb{K}\right)$ with $B_{2}\left(x, x^{*}\right)=\left\langle x, x^{*}\right\rangle$. Here $(\mathcal{B}) \operatorname{var}(f)=\operatorname{var}(f)$.

- $\mathcal{B}_{3}=\left(B_{3} ; L(X, Y), X, Y\right)$ with $B_{3}(T, x)=T x$. Here $(\mathcal{B}) \operatorname{var}(f)$ depends on the spaces $X$ and $Y$.

A look at the definitions of the different 'variations' shows that

$$
\operatorname{semivar}\left(f_{B, y}\right) \leq(\mathcal{B}) \operatorname{var}(f) \leq \operatorname{var}(f)
$$

for every $y \in Y$ with $\|y\|_{Y} \leq 1$ (when $\|B\| \leq 1$ ). In the special case where a Banach space $X$ can be considered to be isometrically embedded in the Banach 
space $L(Y, Z)$ where $Y$ and $Z$ are also Banach spaces, it is not difficult to prove (see [3]) that

$$
\operatorname{semivar}(f) \leq(\mathcal{B}) \operatorname{var}(f) \leq \operatorname{var}(f) .
$$

In [1], dealing with Riemann-Stieltjes integrals and $\mathcal{B}=(B ; L(X), X, X)$ and $B(T, x)=T x$, a question arises. "Is every $L(X)$-valued function with finite $(\mathcal{B})$-variation a $(\mathcal{B})$-regulated function?" The answer to this question is given in the following assertion.

Theorem 1. ([1]) Let $\mathcal{B}=(B ; L(X), X, X)$ and $B(T, x)=T x$. If $X$ is a uniformly convex space then, every $L(X)$-valued function $f$ with $(\mathcal{B}) \operatorname{var}(f)<$ $\infty$ is $(\mathcal{B})$-regulated.

It is clear that every function of bounded variation is $(\mathcal{B})$-regulated for any bilinear map. However, bounded semivariation is not enough to ensure the conclusion.

Our purpose is to improve the result of [1]. The following example has been useful in order to guide our steps. We take the bilinear form $B: \ell^{\infty} \times \ell^{\infty} \rightarrow \ell^{\infty}$ mapping $B\left(\left(\alpha_{n}\right)_{n},\left(\beta_{n}\right)_{n}\right)=\left(\alpha_{n} \beta_{n}\right)_{n}$ and the $\ell^{\infty}$-valued function

$$
f=0 \chi_{\{0\}}+\sum_{k=1}^{\infty} e_{k} \chi_{\left(\frac{1}{k+1}, \frac{1}{k}\right]} .
$$

This function is not $(\mathcal{B})$-regulated but of finite $(\mathcal{B})$-variation.

\section{The Main Theorem}

The reader can deduce from the proofs of our statements that, in spite of using functions, they actually depend upon the behavior of certain telescopic series. In fact, the famous theorem of Bessaga and Pełczinsky is the cornerstone of our results.

Theorem 2 (Bessaga-Pełczynski). (see [2]) The Banach space $X$ contains no copy of $c_{0}$ if and only if all series $\sum_{n} x_{n}$ in $X$, with $\sum_{n}\left|\left\langle x_{n}, x^{*}\right\rangle\right|<\infty$ for all $x^{*} \in X^{*}$, are unconditionally convergent in norm.

Consequently, if $X$ is a Banach space containing a copy of $c_{0}$, we can always find a sequence $\left(x_{n}\right)_{n=1}^{\infty}$ in $X$ and constants $C_{1}, C_{2}>0$ such that for every $N \in \mathbb{N}$

$$
C_{1} \sup _{1 \leq n \leq N}\left|\lambda_{n}\right| \leq\left\|\sum_{n=1}^{N} \lambda_{n} x_{n}\right\|_{X} \leq C_{2} \sup _{1 \leq n \leq N}\left|\lambda_{n}\right| .
$$


Moreover, for such a sequence we have that $\sup _{\left\|x^{*}\right\| \leq 1} \sum_{n=1}^{\infty}\left|\left\langle x_{n}, x^{*}\right\rangle\right|<\infty$ and $\sum_{n=1}^{\infty} x_{n}$ is not norm convergent.

The main theorem is the following.

Theorem 3. Let $Z$ be a Banach space. The following assertions are equivalent:

(1) $Z$ does not contain copy of $c_{0}$.

(2) Every function $f:[0,1] \rightarrow Z$ with finite semivariation is regulated.

Proof. (1) $\Rightarrow(2)$ Let $f$ be a function of finite semivariation. In order to prove that it is regulated at any $t \in[0,1]$, it suffices to show the existence of $\lim _{n} f\left(t_{n}\right)$ for any sequence $\left(t_{n}\right)_{n} \subset[0,1]$ increasing or decreasing to $t$. We shall restrict ourselves to work the case $\left(t_{n}\right)_{n}$ increasing. Let $\left(t_{n}\right)$ be a sequence increasing to $t$. For $N \in \mathbb{N}$ let $d_{N}=\left\{0, t_{1}, t_{2}, \ldots, t_{N}, 1\right\}$ be a partition of $[0,1]$. From the assumptions

$$
\sup _{\left\|z^{*}\right\| \leq 1} \sum_{k=1}^{N}\left|\left\langle f\left(t_{k}\right)-f\left(t_{k-1}\right), z^{*}\right\rangle\right| \leq \operatorname{semivar}(f)<\infty
$$

and therefore $\sup _{\left\|z^{*}\right\| \leq 1} \sum_{k=1}^{\infty}\left|\left\langle f\left(t_{k}\right)-f\left(t_{k-1}\right), z^{*}\right\rangle\right|<\infty$. Making use of the theorem of Bessaga and Pełczynski we have that the telescopic series $\sum_{k=1}^{\infty}\left[f\left(t_{k}\right)-f\left(t_{k-1}\right)\right]$ converges to an element $z \in Z$.

It is easy to prove that the element $z$ is independent of the sequence $\left(t_{n}\right)_{n}$. To that end, choose another sequence $\left(s_{n}\right)_{n}$ increasing to $t$. As above $\sum_{k=1}^{\infty}\left[f\left(s_{k}\right)-f\left(s_{k-1}\right)\right]$ converges to an element $y \in Z$ and therefore $\lim _{n} f\left(t_{n}\right)=z+f(0)$ and $\lim _{n} f\left(s_{n}\right)=y+f(0)$. Finally, consider the sequence $\left(r_{n}\right)_{n}$ made by nondecreasingly ordering the set $\left\{t_{n}: n \in \mathbb{N}\right\} \cup\left\{s_{n}: n \in \mathbb{N}\right\}$. With this new sequence we have that $\sum_{k=1}^{\infty}\left[f\left(r_{k}\right)-f\left(r_{k-1}\right)\right]$ converges to an element $x \in Z$, but as long as it is telescopic, we find in the partial sums of this series, alternatively, subsequences of $\left(f\left(t_{n}\right)\right)_{n}$ and $\left(f\left(s_{n}\right)\right)_{n}$. Then $x=y=z$ and $f$ is regulated.

$(2) \Rightarrow(1)$ Let us assume that $Z$ does contain a copy of $c_{0}$. The theorem of Bessaga and Pełczynski provides us with a sequence $\left(z_{n}\right)_{n} \subset Z$ as the one described in (1). The function $f=0 \chi_{\{0\}}+\sum_{k=1}^{\infty} z_{n} \chi_{\left(\frac{1}{k+1}, \frac{1}{k}\right]}$ defined in $[0,1]$ is not regulated at the origin. Indeed

$$
\left\|f\left(\frac{1}{k}\right)-f\left(\frac{1}{k-1}\right)\right\|=\left\|z_{k}-z_{k-1}\right\| \geq C_{1}
$$

for some $C_{1}>0$, and then the discontinuity of $f$ at $t=0$ is not of first kind. However, if $d \in D$ and $z^{*} \in Z^{*}$

$$
\sum_{k=1}^{|d|}\left|\left\langle f\left(t_{k}\right)-f\left(t_{k-1}\right), z^{*}\right\rangle\right|=\sum_{k=1}^{|d|}\left|\left\langle z_{\left[\frac{1}{t_{k}}\right]}-z_{\left[\frac{1}{t_{k-1}}\right]}, z^{*}\right\rangle\right| \leq 2 \sum_{k=1}^{\infty}\left|\left\langle z_{k}, z^{*}\right\rangle\right|<\infty .
$$


Then semivar $(f)<\infty$ which is a contradiction.

Corollary 4. Let $Z$ be a Banach space. The following assertions are equivalent:

(1) $Z$ does not contain copy of $c_{0}$.

(2) For every $\mathcal{B}=(B ; X, Y, Z)$ where $X$ and $Y$ are Banach spaces and $B: X \times Y \rightarrow Z$ is a bounded bilinear map, we have that every function $f:[0,1] \rightarrow X$ with finite $(\mathcal{B})$-variation is $(\mathcal{B})$-regulated.

Proof. $(1) \Rightarrow(2)$ Given $\mathcal{B}=(B ; X, Y, Z)$ and $f:[0,1] \rightarrow X$ with $(\mathcal{B}) \operatorname{var}(f)<$ $\infty$. Since semivar $\left(f_{B, y}\right)<\infty$ for every $y \in Y$, the previous theorem gives that function $f_{B, y}$ is regulated and therefore $f$ is $(\mathcal{B})$-regulated.

$(2) \Rightarrow(1)$ If $Z$ has a copy of $c_{0}$, we choose $\left(z_{n}\right)_{n} \subset Z$ as it is described in (1). For the situation $\mathcal{B}=(B ; Z, \mathbb{K}, Z)$ we can now use the function defined in the theorem to obtain a contradiction.

Finally, we set the application of this result to the concept of the HenstockKurzweil integral where, for a given Banach space $X$, it is of interest to have functions $f:[0,1] \rightarrow L(X)$ of finite $(\mathcal{B})$-variation and $(\mathcal{B})$-regulated for the particular situation $\mathcal{B}=(B ; L(X), X, X)$ with $B(T, x)=T x$.

Theorem 5. Let $X$ be a Banach space, and let us consider functions $f$ : $[0,1] \rightarrow L(X)$ and the situation $\mathcal{B}=(B ; L(X), X, X)$. The following assertions are equivalent:

(1) $X$ does not contain copy of $c_{0}$

(2) Every function $f:[0,1] \rightarrow L(X)$ with finite $(\mathcal{B})$-variation is $(\mathcal{B})$ regulated.

Proof. (1) $\Rightarrow(2)$ It is an immediate corollary of the previous theorem.

$(2) \Rightarrow(1)$ Let $f:[0,1] \rightarrow L(X)$ be $f=0 \chi_{\{0\}}+\sum_{k=1}^{\infty} \widetilde{T}_{k} \chi_{\left(\frac{1}{k+1}, \frac{1}{k}\right]}$. Operators

$\widetilde{T}_{k}$ are asked to map $X$ on $X$. As long as $X$ has a copy of $c_{0}$ we can fix a sequence $\left(x_{n}\right)_{n}$ as described in (1). Let $Y=\overline{\operatorname{span}}\left\{x_{n}: n \in \mathbb{N}\right\}$ be the copy of $c_{0}$ lying inside $X$, and let $S_{k}: Y \rightarrow \operatorname{span}\left\{x_{2^{k}+n}\right\}_{n=1}^{k}$ be defined by $S_{k}\left(\sum_{n=1}^{\infty} \alpha_{n} x_{n}\right)=\sum_{n=1}^{k} \alpha_{n} x_{2^{k}+n}$. It is easy to check that $S_{k}$ is a continuous linear map and $\left\|S_{k}\right\| \leq \frac{C_{2}}{C_{1}}$ (use (1)). Let $\pi_{j}: Y \rightarrow \mathbb{R}$ be the projection $\pi_{j}\left(\sum_{n=1}^{\infty} \alpha_{n} x_{n}\right)=\alpha_{j}$. It is easy to see that $\left\|\pi_{j}\right\| \leq \frac{1}{C_{1}}$ for every $j \in \mathbb{N}$. Applying the Hahn-Banach Theorem to the compositions $T_{k, j}=\pi_{j} \circ S_{k}$ (each of which has norm at most $\frac{C_{2}}{C_{1}^{2}}$ ) we get extensions $\widetilde{T}_{k, j}: X \rightarrow \mathbb{R}$ with the same norm. Finally, define $\widetilde{T}_{k}: X \rightarrow X$ such that $\widetilde{T}_{k}(x)=\sum_{j=1}^{k} \widetilde{T}_{k, 2^{k}+j}(x) x_{2^{k}+j}$. Then $\widetilde{T}_{k} \in L(X)$ and $\left\|\widetilde{T}_{k}\right\| \leq \frac{C_{2}}{C_{1}^{2}}$. 
Let us show now that $f$ is the desired function. Let us take (for instance) the vector $x_{1}$ of the sequence $\left(x_{n}\right)_{n}$ (normalized if needed) and compute, for $k \in \mathbb{N}$,

$$
\begin{aligned}
\left\|f_{B, x_{1}}\left(\frac{1}{k}\right)-f_{B, x_{1}}\left(\frac{1}{k-1}\right)\right\|_{X} & =\left\|f\left(\frac{1}{k}\right)\left(x_{1}\right)-f\left(\frac{1}{k-1}\right)\left(x_{1}\right)\right\|_{X} \\
& =\left\|\widetilde{T}_{k}\left(x_{1}\right)-\widetilde{T}_{k-1}\left(x_{1}\right)\right\|_{X} \\
& =\left\|x_{2^{k}+1}-x_{2^{k-1}+1}\right\|_{X} \geq C_{1} .
\end{aligned}
$$

Then $f$ is not $(\mathcal{B})$-regulated at $t=0$. Now let $d \in D$ and $w_{k}$ be norm-one elements of $X(k=1, \ldots,|d|)$, denoting by $n_{k}=\left[\frac{1}{t_{k}}\right]$ we have

$$
\begin{aligned}
& \left\|\sum_{k=1}^{|d|}\left[f\left(t_{k}\right)-f\left(t_{k-1}\right)\right]\left(w_{k}\right)\right\|_{X} \leq\left\|\sum_{k=2}^{|d|}\left[\widetilde{T}_{n_{k}}-\widetilde{T}_{n_{k-1}}\right]\left(w_{k}\right)\right\|_{X}+\left\|\widetilde{T}_{n_{1}}\left(w_{1}\right)\right\|_{X} \\
= & \left\|\sum_{k=z}^{|d|} \sum_{j=1}^{n_{k}} \widetilde{T}_{n_{k}, 2^{n_{k}}+j}\left(w_{k}\right) x_{2^{n_{k}+j}} \quad-\sum_{k=2}^{|d|} \sum_{j=1}^{n_{k-1}} \widetilde{T}_{n_{k-1}, 2^{n_{k-1}}+j}\left(w_{k}\right) x_{2^{n_{k-1}}+j}\right\|_{X} \\
& +\left\|\widetilde{T}_{n_{1}}\left(w_{1}\right)\right\|_{X} \leq 2 \frac{C_{2}}{C_{1}^{2}} .
\end{aligned}
$$

Hence $f$ has finite $(\mathcal{B})$-variation.

\section{References}

[1] L. Barbanti, Simply regulated functions and semivariation in uniformly convex spaces, Real Analysis Exchange, Vol. 24(1), 1998/9, 405-410.

[2] J. Diestel-J. J. Uhl, Vector measures, American Mathematical Society, Mathematical Surveys and Monographs, Volume 15, 1977.

[3] N. Dinculeanu, Vector measures, Pergamon Press, International Series of Monographs in Pure and Applied Mathematics, Volume 95, 1967.

[4] C. S. Hönig, Volterra Stieltjes integral equations, Math. Studies 16, N. Holland, Publ. Co., 1975.

[5] J. Kurzweil, Nichtabsolut Konvergente Integrale, BBS B.G. Teubner Verlagsg., Leipzig, 1980. 